\title{
Präzise Befundung mit der OCT-Angiografie - Artefakte erkennen und ausschließen
}

\section{Accurate OCT-angiography Interpretation - Detection and Exclusion of Artifacts}

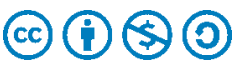

\author{
Autoren \\ Gabriele E. Lang, Christian Enders, Max Loidl, Gerhard K. Lang, Jens Ulrich Werner
}

Institut

Klinik für Augenheilkunde, Universitätsklinikum Ulm

\section{Schlüsselwörter}

OCT-Angiografie, Artefakt, Projektionsartefakt, Fenstereffekt, Abschattungsartefakt

Key words

OCT-angiography, artifact, projection artifact, unmasking, masking

\section{eingereicht 6.4.2017}

akzeptiert 22.5.2017

Bibliografie

DOI https://doi.org/10.1055/s-0043-112857

Online-publiziert 19.6.2017 | Klin Monatsbl Augenheilkd

2017; 234: 1109-1118 @ Georg Thieme Verlag KG Stuttgart .

New York | ISSN 0023-2165

Korrespondenzadresse

Prof. Gabriele E. Lang

Klinik für Augenheilkunde, Universitätsklinikum Ulm

Prittwitzstr. 43, 89075 Ulm

Tel.: + 49/(0)731/50059001, Fax: + 49/(0)731/50059002

gabriele.lang@uniklinik-ulm.de

\section{ZUSAMMENFASSUNG}

Hintergrund Die optische Kohärenztomografie-Angiografie (OCTA) ermöglicht nicht invasiv eine hochaufgelöste und tiefenselektive Visualisierung des Mikrogefäßsystems von Netzhaut und Aderhaut. Allerdings können auch Bildartefakte auftreten, die eine Befundung erschweren. Ziel dieser Arbeit ist es, im Zusammenhang mit OCTA-Aufnahmen auftretende Artefakte zu beschreiben und eine Nomenklatur vorzustellen.

Methoden Es wurden OCTA-Untersuchungen mit der AngioPlex ${ }^{\mathrm{TM}}$-OCTA-Technologie sowie auch mit dem Plex Elite 9000 (Carl Zeiss Meditec, Inc., Dublin, USA) in Kombination mit dem CIRRUS HD-OCT 5000 (Carl Zeiss Meditec, Inc., Dublin, USA) durchgeführt. Beispielhaft werden typische, in den
OCTA-Aufnahmen auftretende Artefakte beschrieben und deren Ursachen erläutert.

Ergebnisse Es können 3 Gruppen von Artefakten unterschieden werden: (a) systemimmanente Artefakte, die unabhängig vom eingesetzten Gerätetyp auftreten (Projektions- und Abschattungsartefakte, Fenstereffekte und der Verlust des Scanfokus); (b) Artefakte, die durch Algorithmen zur Datenverarbeitung und Bildprozessierung hervorgerufen werden und deren Häufigkeit bzw. Ausprägung vom eingesetzten Gerätetyp abhängt und variieren kann (Segmentierungsartefakte, Duplikationen von Gefäßen); (c) Bewegungsartefakte, die je nach eingesetztem Gerätetyp in unterschiedlicher Häufigkeit und Ausprägung auftreten, da je nach Gerät unterschiedliche Methoden (z. B. leistungsfähiger Eye-Tracker) eingesetzt werden, um diese zu reduzieren. Das Auftreten von Artefakten ist außerdem auch abhängig von der Kooperation der Patienten, der Klarheit der optischen Medien und der Pathologie der Netzhaut.

Schlussfolgerung Bei der OCTA treten wie bei jedem anderen bildgebenden Verfahren auch Artefakte auf. Dennoch lassen sich die OCTA-Aufnahmen qualitativ fast immer gut auswerten und liefern unverzichtbare Erkenntnisse zu Morphologie und Perfusionsstatus von Aderhaut und Netzhaut. Bei guter Kenntnis möglicher Artefakte und entsprechender kritischer Datenanalyse ist eine korrekte Bewertung der OCTAAufnahmen möglich, um eine korrekte klinische Diagnose zu stellen.

\section{ABSTRACT}

Background Optical coherence tomography angiography (OCTA) provides, non-invasively, a three-dimensional visualization of the microvasculature of the retina and choroid. However, image artifacts may occur in OCTA and have an impact on clinical interpretation. The aim of this article is to describe image artifacts of OCTA and to present a nomenclature.

Methods OCTA examinations were performed with the AngioPlex ${ }^{\mathrm{TM}}$ OCTA-technology in combination with the CIRRUS HD-OCT 5000 (Carl Zeiss Meditec, Inc., Dublin, USA) as well as with the PlexElite 9000 (Carl Zeiss Meditec, Inc., Dublin, 
USA). Typical artifacts identified in the OCTA images are described and their causes are explained.

Results There are three main groups of artifacts that can be distinguished: (a) artifacts that are inherent in the OCTA technology and occur with all types of devices (projection artifacts, masking, unmasking, loss of signal); (b) artifacts caused by data and image processing algorithms and whose frequency or severity may depend on the device type used (segmentation artifacts, duplications of vessels); (c) motion artifacts that vary in frequency and severity depending on the type of device used, as different methods (e.g., eye tracker) are used to reduce them. The occurrence of artifacts is also dependent on patient cooperation, the clarity of the optical media, and the pathology of the retina.

Conclusion As in any other imaging method, artifacts also occur in OCTA images. Nevertheless, qualitative assessment of OCTA images is almost always possible and provides indispensable findings on the morphology and perfusion status of the retina and choroid. A good knowledge of possible artifacts, and a critical analysis of the complete OCTA data set, allows a correct interpretation and is essential for making a precise clinical diagnosis.

\section{Einleitung}

Mit der Einführung der optischen Kohärenztomografie-Angiografie (OCTA) in den klinischen Alltag hat vor 2 Jahren eine neue Ära in der Retinadiagnostik begonnen. Mit diesem hochinteressanten Bildgebungsverfahren, das eine funktionale Erweiterung des strukturellen, intensitätsbasierten OCTs darstellt, kann nun in nur wenigen Sekunden nicht invasiv das Mikrogefäßsystem der Netzhaut und Aderhaut hochaufgelöst und tiefenselektiv dargestellt werden. Dies erleichtert die exakte Diagnose und die Verlaufskontrolle vaskulärer Erkrankungen des hinteren Augenabschnitts, da wesentliche Erkenntnisse sowohl zur Morphologie als auch zum Perfusionsstatus gleichzeitig und nicht invasiv gewonnen werden. Dementsprechend hat diese Technologie ein sehr großes Potenzial hinsichtlich Diagnose und Verlaufskontrolle verschiedener retinaler Erkrankungen wie z. B. diabetischer Retinopathie (DR), altersbezogener Makuladegeneration (AMD), retinaler Gefäßverschlüsse (RVV) und Glaukom, wie zahlreiche Arbeiten belegen [1-3]. Die OCTA liefert sehr detailreiche, 3-dimensionale Darstellungen des gesamten Mikrogefäßsystems von Netz- und Aderhaut. Alle mikrovaskulären Veränderungen verschiedener Erkrankungen wie Mikroaneurysmen, intraretinale mikrovaskuläre Anomalien, nicht perfundierte Areale, Veränderung der foveal-avaskulären Zone (FAZ) und Neovaskularisationen können zuverlässig dargestellt werden. Erste Ergebnisse sind bereits veröffentlicht worden [1].

Gleichwohl können - wie generell bei jedem bildgebenden Verfahren - auch bei der OCTA Bildartefakte auftreten [4-9]. Diese können im Rahmen von Datenerfassung bzw. Bildprozessierung entstehen, auf Besonderheiten okulärer Strukturen zurückzuführen sein oder durch Augen- bzw. Körperbewegungen während des Messvorgangs hervorgerufen werden. Grundsätzlich kann es sich bei Artefakten um Strukturen handeln, die nicht real sind, fehlen oder die am falschen Ort zu sein scheinen, sowie Darstellungen in falscher Helligkeit, Größe oder Form.

Werden sie nicht zuverlässig als Artefakt erkannt, so können sie zu klinischen Fehlinterpretationen führen. Um dies zu vermeiden, gilt es daher, bei der Interpretation der Ergebnisse bildgebender Verfahren generell zunächst sicherzustellen, dass die gewonnene Bildinformation nicht auf Artefakte zurückzuführen ist, sondern tatsächlich die Realität abbildet. Um OCTA-Aufnahmen klinisch korrekt interpretieren und bewerten zu können, ist daher - neben einer genauen Kenntnis der Netzhautmorphologie - auch ein gutes Verständnis der Entstehung und Auswirkung möglicher Artefakte unerlässlich.

Ziel der vorliegenden Arbeit ist es, typische, im Zusammenhang mit OCTA-Aufnahmen auftretende Artefakte hinsichtlich ihrer Entstehung und Ausprägung zu beschreiben und eine Nomenklatur vorzustellen.

\section{Methoden}

Seit Oktober 2015 verwenden wir die AngioPlex ${ }^{\mathrm{TM}}$-OCTA-Technologie von Zeiss in Kombination mit dem Spectral Domain OCT CIRRUS HD-OCT 5000 (Carl Zeiss Meditec, Inc., Dublin, USA) zur Diagnostik von Netzhaut-Aderhaut-Erkrankungen und haben kürzlich auch mit dem Swept-Source-OCT-basierten PlexElite 9000 (Carl Zeiss Meditec, Inc., Dublin, USA) Untersuchungen durchgeführt. Mit der Angioplex-OCTA können Volumenscans der Größen $8 \times 8,6 \times 6$ und $3 \times 3 \mathrm{~mm}$ aufgenommen werden, während mit dem PlexElite Modell auch Volumenscans von $12 \times 12$ mm erstellt werden können. Die 3×3-mm-Volumenscans liefern die höchste Auflösung. Das CIRRUS HD-OCT 5000 arbeitet mit Scangeschwindigkeiten von bis zu $68 \mathrm{KHz}$ und ist mit einem Zeilenscanophthalmoskop (LSO) ausgestattet. Mithilfe des FastTrac $^{\mathrm{TM}}$-Eye-Tracking-Systems können auch kleine Augenbewegungen zuverlässig nachverfolgt werden. Dies sorgt für ein sehr kontrastreiches Bild und eine gute Überlagerung verschiedener Scans eines Auges, was zu einer Reduktion von Bewegungsartefakten beiträgt. Um den Blutfluss anhand von zeitlichen Kontrastunterschieden der gleichen Lokalisation zu ermitteln, werden in der AngioPlex-OCTA sog. OMAGc-Algorithmen (OMAGc: „Optical Micro Angiography Complex") eingesetzt, die sowohl Amplituden- als auch Phasensignale der B-Scans vergleichend auswerten und so zu einer hohen Bildqualität beitragen [10].

Auch wenn die Ursachen verschiedener Artefakte nicht in allen technischen Details beschrieben werden sollen, so ist es doch zur klinischen Bewertung der OCTA-Aufnahmen wichtig, grundsätzlich das Messprinzip der OCTA zu verstehen. Prinzipiell werden in der OCTA zeitliche Änderungen im Reflexionsverhalten von Gewebeschichten detektiert und diese einem Blutfluss zugeordnet. Zur Erfassung dieser zeitlichen Kontrastveränderung werden zunächst mehrfach kurz hintereinander an einer Stelle der Netzhaut OCTSchnittbilder (B-Scans) aufgenommen. Anschließend werden alle OCT-Schnittbilder einer Lokalisation mithilfe spezieller Algorithmen vergleichend ausgewertet. Es ergeben sich Bereiche, in denen eine zeitliche Veränderung des Kontrasts auftritt. Diese wer- 
- Tab. 1 Typische, in der OCTA auftretende Artefakte und ihre Definitionen.

\begin{tabular}{|c|c|c|}
\hline & Artefakt & Definition \\
\hline \multirow{4}{*}{$\begin{array}{l}\text { system- } \\
\text { immanente } \\
\text { Artefakte } \\
\text { der OCTA }\end{array}$} & Projektionsartefakt (projection artifact) & $\begin{array}{l}\text { Gefäßstrukturen oberflächlicher Schichten werden fälschlicherweise } \\
\text { auch in tiefer gelegenen Schichten dargestellt. }\end{array}$ \\
\hline & Abschattungsartefakt (masking) & $\begin{array}{l}\text { Dichte Medien können zu Signalverlust in darunter liegenden Schichten } \\
\text { führen und deren Visualisierung erschweren. }\end{array}$ \\
\hline & Fenstereffekt (unmasking) & $\begin{array}{l}\text { Ausfall bestimmter Bereiche (z. B. Atrophie) führt zu Signalverstärkung } \\
\text { in darunter liegenden Schichten. }\end{array}$ \\
\hline & Verlust des Scanfokus (loss of signal) & $\begin{array}{l}\text { Verlust des Scanfokus in bestimmten Netzhautbereichen, z. B. durch } \\
\text { Tumor, hohe Myopie. Gut erkennbar im OCT-Schnittbild. }\end{array}$ \\
\hline \multirow{2}{*}{$\begin{array}{l}\text { Artefakte } \\
\text { durch Bild- } \\
\text { prozessierung }\end{array}$} & Segmentierungsartefakt (segmentation artifact) & $\begin{array}{l}\text { Fehler in der (automatischen) Segmentierung kann zu auffälligen } \\
\text { OCTA-Befunden führen. }\end{array}$ \\
\hline & Duplikation von Gefäßen (duplication of vessels) & $\begin{array}{l}\text { Gefäße werden infolge von Bildprozessierung innerhalb einer Schicht } \\
\text { direkt nebeneinander doppelt dargestellt. }\end{array}$ \\
\hline \multirow[t]{4}{*}{$\begin{array}{l}\text { Artefakte } \\
\text { durch } \\
\text { Bewegung }\end{array}$} & Bewegungsartefakt (motion artifact) & $\begin{array}{l}\text { Sehr dünne weiße, horizontale Linien, die zu einer scheinbaren Unter- } \\
\text { brechung oder Verschiebung der Gefäße führen, hervorgerufen durch } \\
\text { Augenbewegungen. }\end{array}$ \\
\hline & Blinzelartefakt (blink artifact) & $\begin{array}{l}\text { Vertikale und horizontale schwarze Linien in OCTA-Aufnahmen jeder } \\
\text { Schicht, hervorgerufen durch Blinzeln. }\end{array}$ \\
\hline & Bandenbildung (banding) & $\begin{array}{l}\text { Nebeneinander liegende horizontale Streifen unterschiedlicher Hellig- } \\
\text { keit. }\end{array}$ \\
\hline & $\begin{array}{l}\text { Randduplikation/Stretch-Artefakt (edge duplication/ } \\
\text { stretch artifact) }\end{array}$ & $\begin{array}{l}\text { Kurze Streifen unterschiedlicher Helligkeit im Randbereich von OCTA- } \\
\text { Aufnahmen. }\end{array}$ \\
\hline
\end{tabular}

den einem Blutfluss, d. h. einem perfundierten Gefäß zugeordnet. Bereiche, in denen keine zeitliche Kontraständerung detektiert werden kann, werden dem umliegenden Gewebe zugeordnet. Insgesamt wird so das gesamte Mikrogefäßsystem von Netzhaut und Aderhaut innerhalb eines Volumenscans 3-dimensional erfasst. Durch eine automatische Segmentierung wird es anschließend gewissermaßen „schichtweise“ in mehreren Projektionsdarstellungen (im Folgenden als OCTA-Aufnahmen bzw. OCTA-Bild bezeichnet) visualisiert. Dazu werden - basierend auf dem gesamten Datensatz eines Volumenscans - softwaregesteuert automatisch spezifische Schichten der Netzhaut bzw. Aderhaut ausgewählt. Welche Schicht in einer bestimmten OCTA-Aufnahme dargestellt wird, zeigen jeweils die Segmentierungslinien im korrespondierenden OCT-Schnittbild an. Innerhalb dieses klar begrenzten Bereichs werden alle Signale entlang der Richtung des OCT Strahls quasi aufsummiert, sodass in einer OCTA-Aufnahme alle erfassten Gefäße innerhalb der ausgewählten Schicht dargestellt werden. Automatisch werden voreingestellt OCTA-Aufnahmen der vitreoretinalen Grenzschicht, der gesamten neurosensorischen Netzhaut, der Choriokapillaris und der Aderhaut erstellt. Zur detaillierten Darstellung der retinalen Gefäße werden zudem OCTA-Aufnahmen einzelner Schichten der neurosensorischen Netzhaut (oberflächlicher Gefäßplexus, tiefer Gefäßplexus und avaskuläre Retina) erstellt.

Wichtig für die korrekte Interpretation ist die Kenntnis von möglichen Artefakten. Einige Artefakte sind bereits von strukturellen OCT-Untersuchungen oder Laser-Scanning-Geräten her bekannt. Bei der Erfassung und Beschreibung der in den OCTA-Bildern identifizierten Artefakte haben wir uns an bereits publizierten Kategorien orientiert [5, 7].

\section{Ergebnisse}

Im Folgenden werden typische, in OCTA-Aufnahmen identifizierte Artefakte beschrieben und deren Ursachen erläutert. Um eine Zuordnung zur englischsprachigen Nomenklatur zu ermöglichen, wird jeweils auch die englische Bezeichnung in Klammern aufgeführt. Eine tabellarische Übersicht der Artefakte einschließlich ihrer Definition ist in $>$ Tab. 1 dargestellt.

Hinsichtlich der OCTA können im Wesentlichen 3 Gruppen von Artefakten unterschieden werden:

1. Artefakte, die der Methode an sich geschuldet sind und die daher unabhängig vom eingesetzten Gerätetyp auftreten. Hierzu zählen Projektions- und Abschattungsartefakte ebenso wie Fenstereffekte und der Verlust des Scanfokus.

2. Artefakte, die durch Algorithmen zur Datenverarbeitung und Bildprozessierung hervorgerufen werden und deren Häufigkeit bzw. Ausprägung daher vom eingesetzten Gerätetyp abhängt und variieren kann. Hierzu zählen Segmentierungsartefakte und Duplikationen von Gefäßen.

3. Artefakte, die durch Bewegungen hervorgerufen werden. Auch diese können je nach eingesetztem Gerätetyp in unterschiedlicher Häufigkeit und Ausprägung auftreten, da je nach Gerät unterschiedliche Methoden (z. B. leistungsfähiger EyeTracker) eingesetzt werden, um diese zu reduzieren. Nicht zuletzt sind die Artefakte auch abhängig von der Kooperation der Patienten, der Klarheit der optischen Medien und der Pathologie der Netzhaut. 

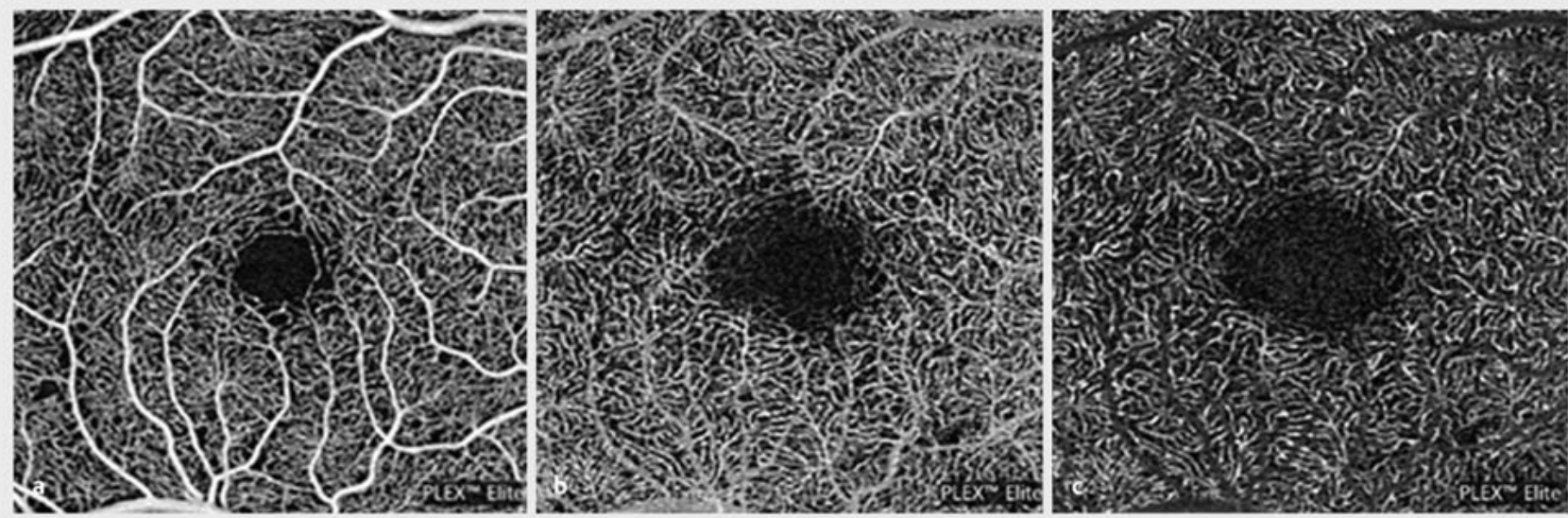

- Abb. 1 Das OCTA einer normalen Makula mit Swept-Source-Technologie (Plex SS Elite Zeiss) zeigt den oberflächlichen Gefäßplexus (a), der tiefe Gefäßplexus weist ohne Nachbearbeitung Projektionsartefakte von oberflächlichen Gefäßen auf (b). Die Projektionsartefakte können mit einem speziellen Programm entfernt werden und es zeigen sich dann die Bereiche der projizierten Gefäße dunkel (c).

\section{Systemimmanente Artefakte der OCTA}

Projektionsartefakt (projection artifact)

Wie bereits beschrieben, stellt die OCTA zeitliche Änderungen im Reflexionsverhalten von Gewebeschichten dar. Allerdings kann der Blutfluss in einem oberflächlichen Gefäß auch eine Signalveränderung in einer tiefer liegenden Gewebestruktur hervorrufen. Dies geschieht, weil der Blutfluss in einem oberflächlichen Gefäß auch in darunter liegenden Schichten zu zeitlich veränderlichen Abschattungen führen kann. Diese Signalveränderung in der tieferen Schicht wird vom Gerät ebenfalls als Blutfluss erfasst, sodass die oberflächliche Gefäßstruktur - fälschlicherweise - als eine Projektion in den tieferen Gewebeschichten erscheint. Die Folge ist, dass oberflächlich gelegene Gefäße zusätzlich auch in OCTA-Aufnahmen tiefer gelegener Schichten dargestellt werden können. So können im tiefen Gefäßplexus etwa Gefäßnetze des oberflächlichen Gefäßplexus erscheinen ( $\bullet \mathbf{A b b} .1 \mathbf{a}-\mathbf{c}$ ). Durch Algorithmen können Projektionsartefakte entfernt werden, die vorher projizierten Gefäße sind dann häufig als „Negativ“ weiterhin erkennbar, da unter den oberflächlichen Gefäßen im tiefen Gefäßplexus keine Blutflussregistrierung erfolgt ( $\bullet \mathbf{A b b} \mathbf{1} \mathbf{1}$ b, c). Es können vor allem auch in stark hyperreflektiven Schichten wie dem retinalen Pigmentepithel (RPE) fälschlicherweise Gefäße erscheinen, die eigentlich höher liegen. In der Regel lassen sich Projektionsartefakte bei sorgä̈ltiger Analyse des gesamten Datensatzes leicht identifizieren. Treten charakteristische Gefäßverläufe in mehreren Schichten gleichzeitig auf, so ist das ein starker Hinweis auf ein Projektionsartefakt. Im B-Scan mit Darstellung der Blutflussregistrierung erkennt man den Blutfluss in der oberflächlichen Netzhaut und dessen Projektion bis in die tiefen Schichten ( Abb. 2).

\section{Abschattungsartefakt (masking artifact)}

Die in der OCTA gemessene zeitliche Kontrastveränderung zur Detektion eines Blutflusses hängt zudem auch unmittelbar von der Gesamtmenge des Lichtes ab, das auf die zu untersuchende Struktur fällt. Denn es gilt: Je stärker die absoluten zeitlichen Ver-

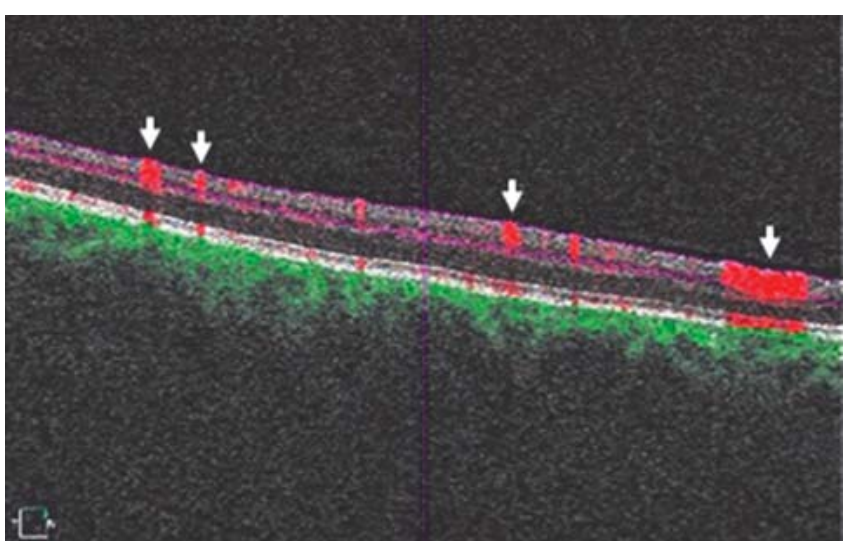

- Abb. 2 In dem B-Scan-Durchlauf einer normalen Netzhaut sieht man rot farbcodiert die Registrierung des Blutflusses in den oberflächlichen Gefäßen, die bis in die unteren Schichten projiziert werden (Pfeile).

änderungen des Reflexionssignals sind, desto stärker ist der Bildkontrast an dieser Stelle. Wird die absolute Lichtmenge durch vorgelagerte, eingetrübte Medien reduziert, so kann dies zu Signalausfällen führen. So können bspw. Glaskörper-Floater, eine mature Katarakt oder Glaskörperblutungen ( $\bullet$ Abb. 3) Abschattungsartefakte in den Netzhautschichten hervorrufen. Subretinale Blutungen können die Visualisierung von Gefäßen in der Choriokapillaris und in der Aderhaut beeinträchtigen. Auch sehr stark reflektive Schichten wie z. B. das RPE oder Fibrosen oder auch sehr dicke Schichten (z. B. durch Ödeme) können durch Abschattung die Visualisierung der darunter liegenden Schichten erschweren. Sehr großflächige Eintrübungen, wie z.B. ein ausgeprägtes Ödem, können eine Art „Rauschen“ hervorrufen. In einzelnen oder sämtlichen Schichten können graue Punktwolken auftreten, und die Schichten erscheinen wie verwaschen, oder es zeigt sich eine schwarze Fläche. Die großen Gefäße sind meistens noch dar- 

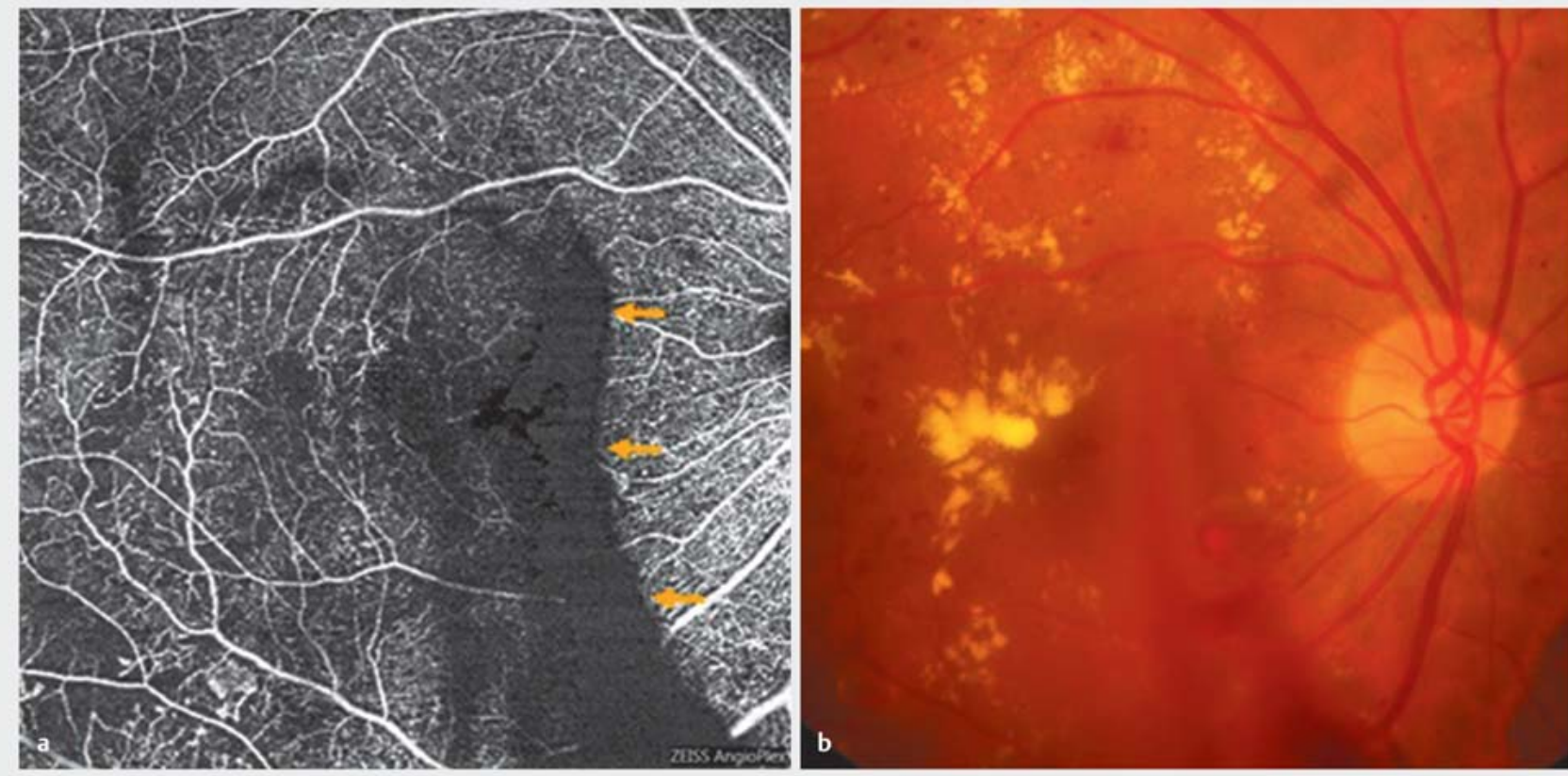

- Abb. 3 Bei einem Patienten mit proliferativer diabetischer Retinopathie zeigt sich im Bereich der Glaskörperblutung in der OCTA ein Abschattungsartefakt (a, Pfeile), korrespondierendes klinisches Bild (b).
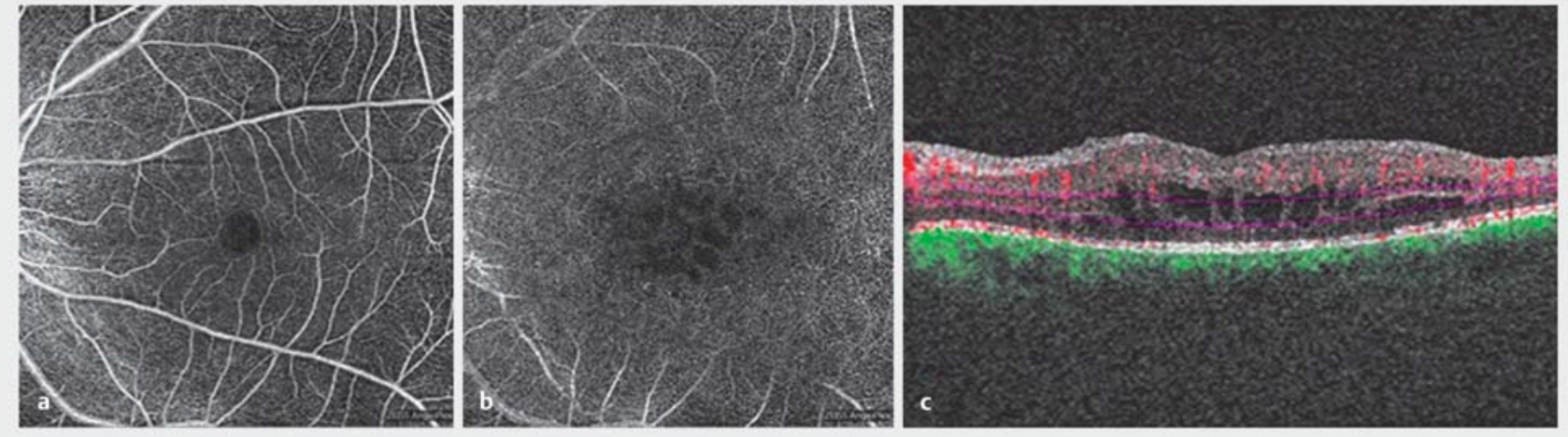

- Abb.4 Abschattungseffekt durch eine zystoide Makulopathie, der Gefäßplexus wird regelrecht dargestellt (a), im tiefen Gefäßplexus sieht man Abschattungen durch das Makulaödem (b), die zystoide Makulopathie stellt sich auch im B-Scan dar (c).

gestellt. Beispielhaft ist hier ein Patient mit zystoidem, diabetischem Makulaödem dargestellt ( $\triangleright$ Abb. 4 a-c).

\section{Fenstereffekt (unmasking artifact)}

Umgekehrt ermöglicht ein Wegfall oberflächlicher Schichten ein besseres Eindringen des OCT-Strahles in die tiefer gelegenen Schichten. Dies kann zu einem stärkeren Bildkontrast führen, sodass an dieser Stelle die normale Gefäßperfusion im Vergleich zum umliegenden Gewebe besser detektiert werden kann. So kann z. B. in Augen mit geografischer Atrophie das Licht durch die atrophischen RPE-Bereiche deutlich besser in die darunter ge- legenen Schichten eindringen als in den nicht atrophischen Bereichen. Dadurch wird die Perfusion der Aderhaut direkt unter den atrophischen Bereichen des RPE besser detektiert als die Aderhautperfusion im umliegenden Gewebe und könnte daher fälschlicherweise - für eine Neovaskularisation gehalten werden. Eine sorgfältige Analyse des korrespondierenden OCT-Schnittbilds sowie der OCTA-Aufnahmen aller Schichten ermöglicht allerdings i.d.R. eine schnelle Identifizierung von Artefakten durch Fenstereffekte ( $\triangleright$ Abb. 5 a, b). 

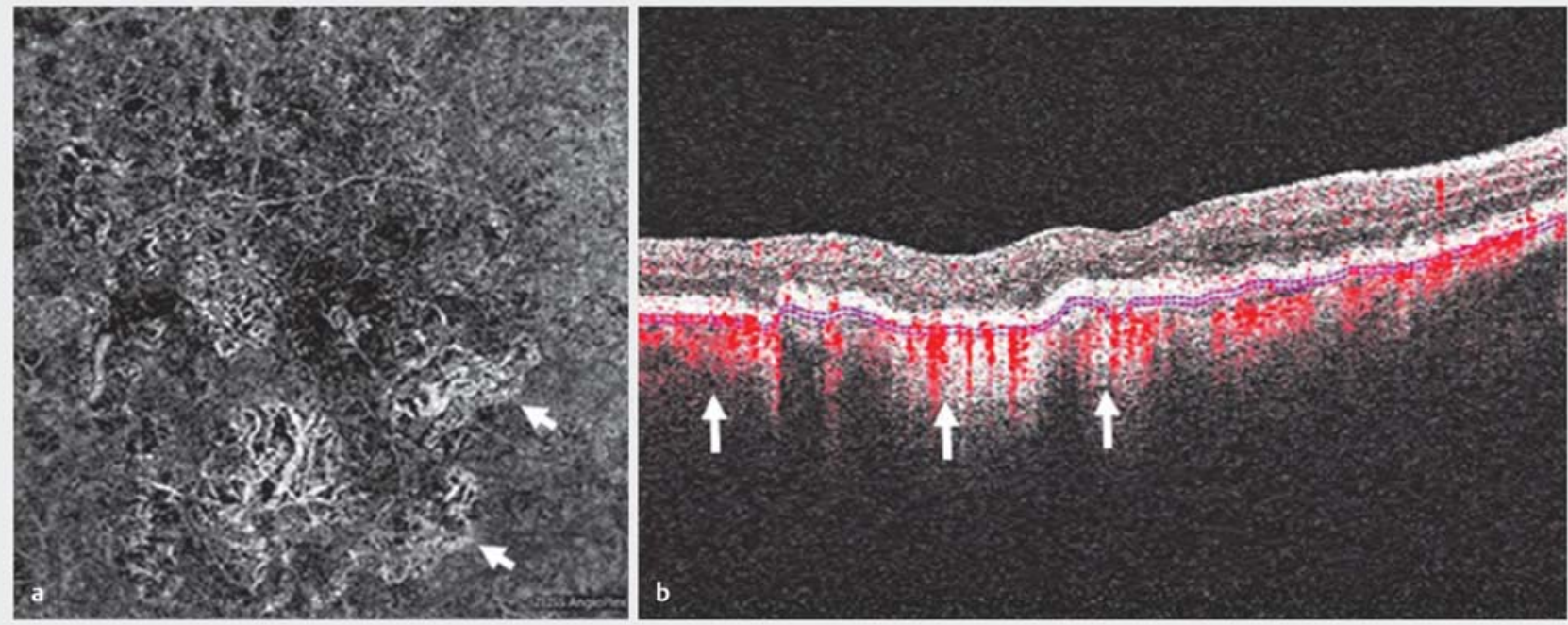

- Abb. 5 Der Fenstereffekt bei einem Patienten mit geografischer Atrophie zeigt eine deutliche Darstellung von Aderhautgefäßen, die mit einer chorioidalen Neovaskularisation verwechselt werden können (a). Der korrespondierende B-Scan zeigt die Blutflussregistrierung der Aderhaut (b, rot farbcodiert, Pfeile).
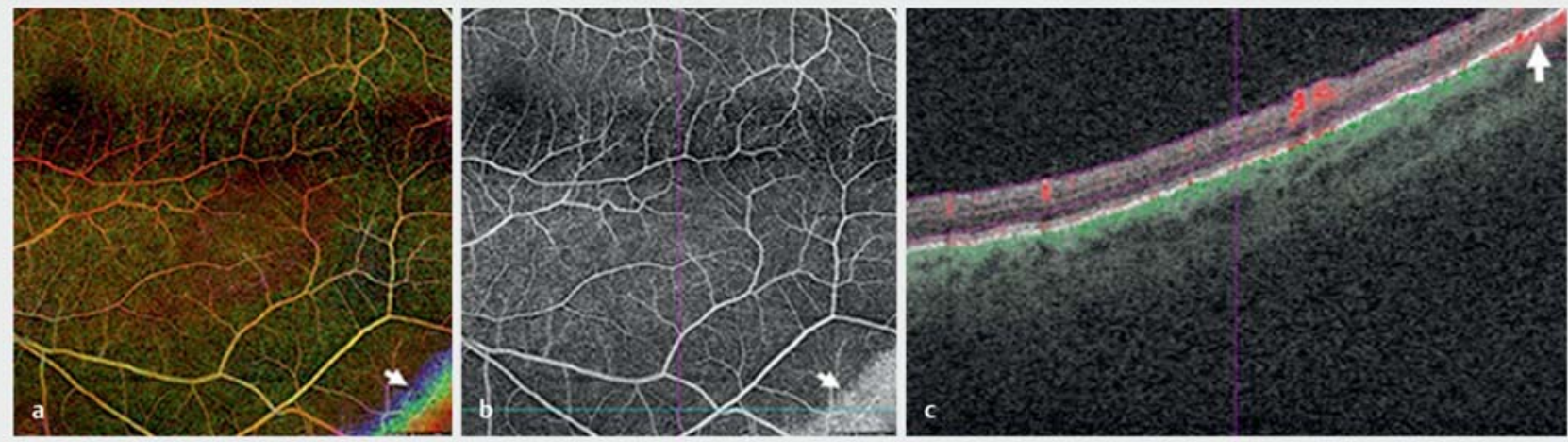

- Abb. 6 Verlust des Scanfokus in der farbcodierten Retinatiefendarstellung in Regenbogenfarben (Pfeil, a), in der Retinasegmentierung (Pfeil, b) und im B-Scan (Pfeil, c).

Verlust des Scanfokus (loss of signal)

Dieses Artefakt ist bereits von strukturellen OCT-Untersuchungen her bekannt. Es tritt auf, wenn sich Teile der Netzhaut durch mangelnde Fokussierung oder die Konfiguration des hinteren Augenabschnitts (z. B. Staphylom bei hoher Myopie) nicht mehr innerhalb des Scanausschnitts befinden. Hervorgerufen werden kann dies u. a. auch durch Tumoren oder ein ausgeprägtes Netzhautödem. Durch diese Gewebsstrukturen kommt es zu einer abrupten Verschiebung der Netzhautebene entlang der z-Achse, sodass B-Scans nicht mehr die Netzhaut erfassen. In den OCTA-Aufnahmen erscheinen diese Bereiche weiß oder schwarz. Meist sind die Areale deutlich heller oder dunkler als normal oder nicht perfundierte Bereiche und daher gut von diesen zu unterscheiden. Im korrespondierenden OCT-Schnittbild lassen sich diese Artefakte anhand der Segmentierungslinien und der Verlust des Scans sofort erkennen. In der farbcodierten OCTA-Darstellung der gesamten Netzhaut stellt sich dieses Artefakt typischerweise als regenbogenartige Struktur im Randbereich dar ( $\boldsymbol{A} \mathbf{A b b} \mathbf{6} \mathbf{6} \mathbf{a}-\mathbf{c}$ ). Tritt ein derartiges Artefakt auf, so empfiehlt es sich, einen kleineren Scanausschnitt (z.B. $3 \times 3$ oder $6 \times 6 \mathrm{~mm}$ ) zu wählen, um für eine durchgängig korrekte Fokussierung zu sorgen.

\section{Artefakte durch Bildprozessierung}

Segmentierungsartefakt (segmentation artifact)

Die automatisch vom System erstellten Segmentierungen nehmen Bezug auf Lage und Reflektivität typischer Netzhautschichten im gesunden Auge. Insbesondere durch ausgeprägte pathologische Veränderungen der Netzhaut oder Aderhaut kann es zu 


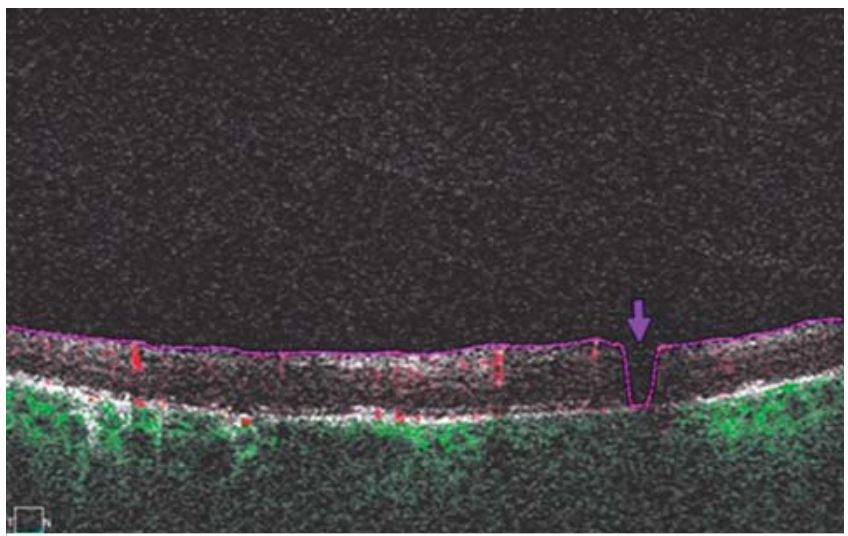

- Abb. 7 Segmentierungsartefakt bei einem B-Scan mit schwacher Reflexion (Pfeil).

Abweichungen vom Normalbefund kommen. Dementsprechend können typische reflektive Schichten, die zur automatischen Segmentierung benötigt werden, nicht mehr zuverlässig vom Gerät erkannt werden. Dies kann zu fehlerhafter automatischer Segmentierung führen und scheinbar auffällige OCTA-Aufnahmen zur Folge haben. So können z. B. eine ausgeprägte RPE-Abhebung durch Drusen oder Fibrosierung sowie auch ein stark ausgeprägtes Ödem dazu führen, dass typische Strukturen von der Software nicht mehr korrekt erkannt und die automatischen Segmentierungslinien daher fehlerhaft gesetzt werden. Auch geringe Reflexion kann zu fehlerhafter Segmentierung führen ( $\triangleright$ Abb. 7). Ein Hinweis auf eine falsche Segmentierung können z.B. schwarze Flecken im korrespondierenden OCTA-Bild sein ( $\bullet$ Abb. 8). Auch wenn die Segmentierungslinien sehr nahe beieinander liegen oder sich gar überkreuzen, werden häufig schwarze Areale gefunden.
Es gilt daher immer, die gewählten Segmentierungslinien im korrespondierenden OCT-Schnittbild auf Plausibilität zu prüfen und ggf. Anpassungen der Segmentierung vorzunehmen, um eine korrekte Interpretation zu gewährleisten. Werden Erkrankungen im zeitlichen Verlauf kontrolliert, so ist es außerdem wichtig, dass die Segmentierungslinien jeweils an der gleichen Stelle liegen. Bei dem hier verwendeten Gerät lassen sich Segmentierungsfehler manuell korrigieren.

\section{Duplikation von Gefäßen (doubling of retinal vessels)}

Dieses Artefakt wird durch Aufnahme- und Bildprozessierungsvorgänge zum Ausgleich von Bewegungsartefakten hervorgerufen. So werden bei Augenbewegungen bestimmte Bereiche häufiger untersucht und eine anschließende Bildnachbearbeitung durchgeführt, um Bewegungsartefakte zu vermeiden. In bestimmten Fällen kann dies dazu führen, dass im OCTA-Bild die Gefäße scheinbar doppelt direkt nebeneinander versetzt erscheinen ( $>$ Abb. 9). Diese charakteristische Verdopplung der Gefäße kann in OCTA-Aufnahmen aller Schichten von Netzhaut und Aderhaut auftreten und lässt sich i. d. R. meist schnell als Artefakt erkennen.

\section{Artefakte, die durch Bewegungen hervorgerufen werden}

Bewegungsartefakt (motion artifact)

Wie bereits beschrieben, werden in der OCTA zeitliche Kontrastveränderung detektiert und diese einem Blutfluss zugeordnet. Dazu werden kurz hintereinander mehrere OCT-Schnittbilder an einer Stelle der Netzhaut aufgenommen und hinsichtlich ihres Kontrasts verglichen. Um dies zu ermöglichen, müssen die aufeinanderfolgenden B-Scans tatsächlich an exakt der gleichen Stelle aufgenommen werden. Je nach Hersteller sorgen verschiedene Strategien wie z. B. ein leistungsfähiger Eye-Tracker grundsätzlich für eine sehr gute Überlagerung der B-Scans. Dennoch
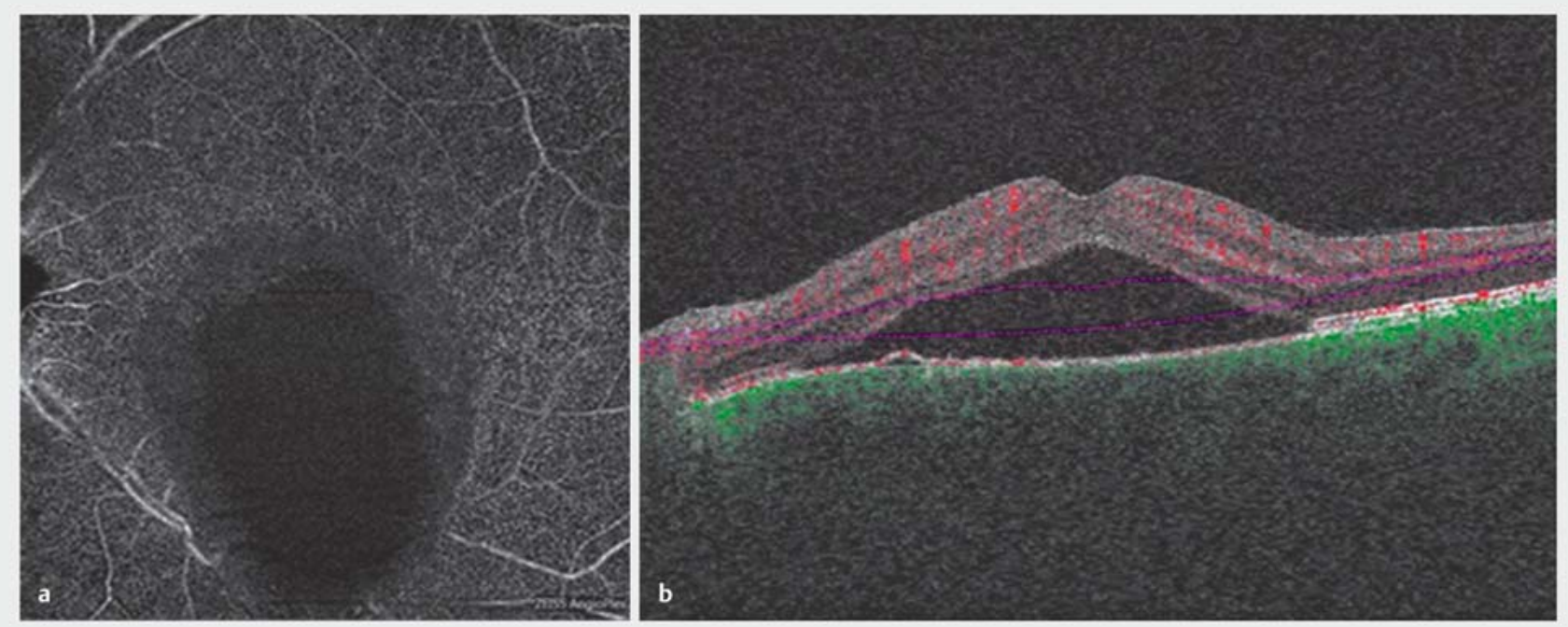

- Abb. 8 Segmentierungsartefakt bei einem ausgeprägten Makulaödem, das zu einer Abschattung im tiefen Gefäßplexus führt (a) und im B-Scan den Segmentierungsfehler zeigt (b). 

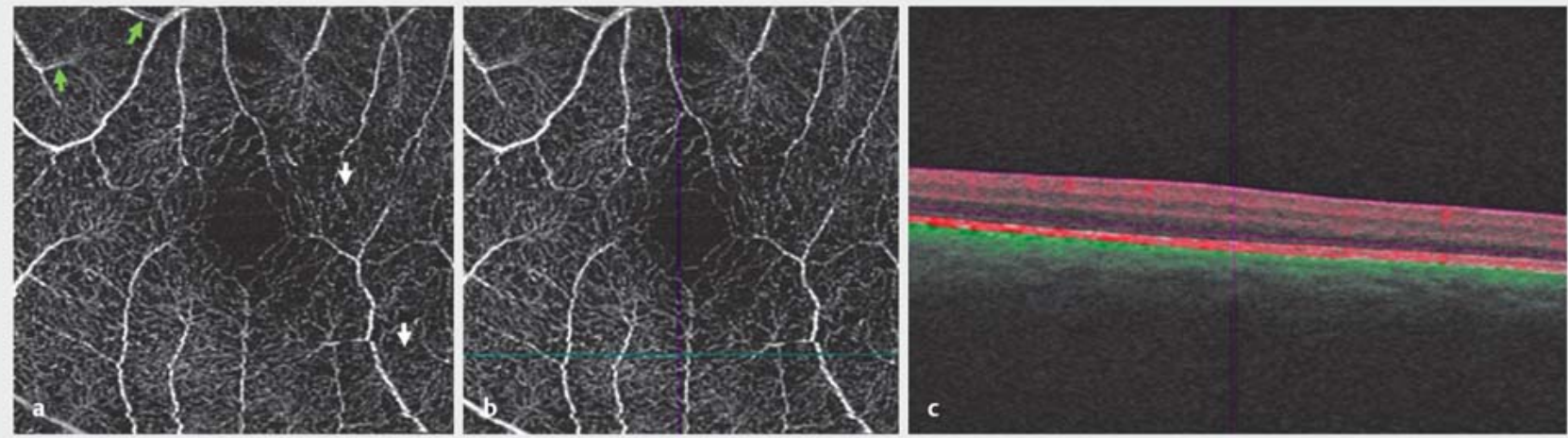

- Abb. 9 Bei reduzierter Kooperation des Patienten zeigen sich Blinzelartefakte (a, weiße Pfeile) und Gefäßduplikationen (grüne Pfeile), Blinzelartefakt mit Navigationslinien (b) und im B-Scan (c).

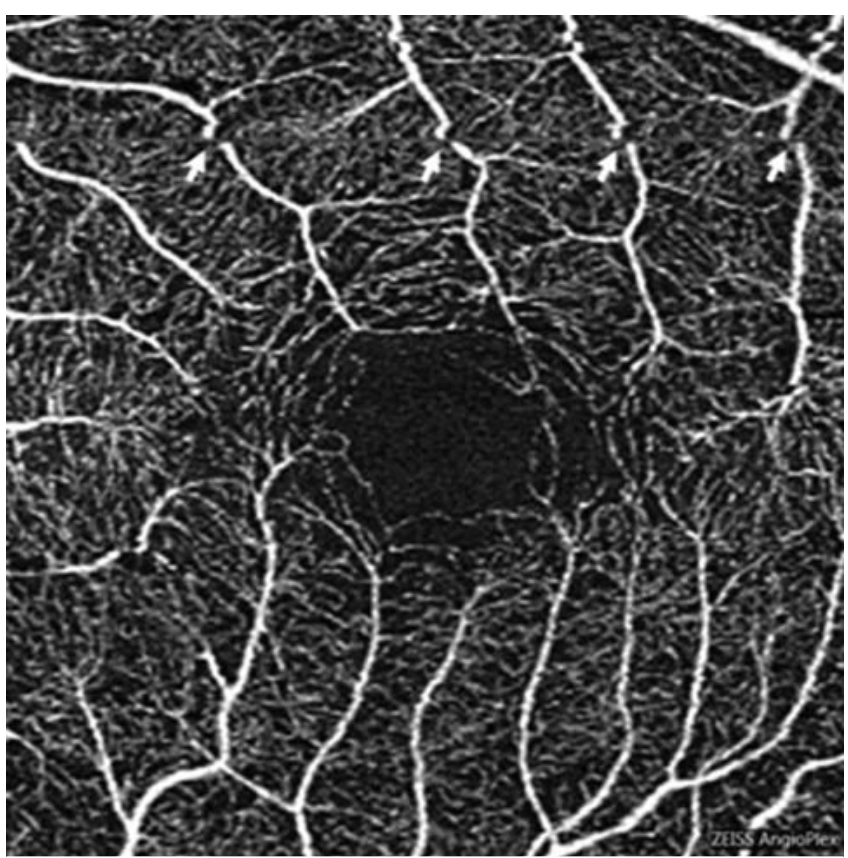

- Abb. 10 Bewegungsartefakte mit weißen Linien und horizontaler Verschiebung von Gefäßen (Pfeile).

können Bewegungen des Patienten oder minimale Augenbewegung dazu führen, dass 2 direkt aufeinanderfolgende B-Scans nicht mehr an der gleichen Position erfasst werden. Sie lassen sich daher nicht mehr überlagern, an dieser Stelle entfällt das Signal und letztlich zeigt sich dies im OCTA-Bild als eine dünne, horizontale weiße oder schwarze Linie. An diesen Streifen wirken die Gefäße scheinbar unterbrochen oder können seitlich versetzt erscheinen ( $\mathbf{A b b} \cdot \mathbf{9 a}-\mathbf{c}, \mathbf{1 0}, \mathbf{1 1}$ ). Bei massiver Ausprägung kann dies die Interpretation der OCTA-Aufnahme auch erschweren oder selten unmöglich machen. In den allermeisten Fällen kann jedoch der Gefäßverlauf auch bei Vorhandensein von Bewegungsartefakten gut identifiziert werden. Insbesondere bei Patienten

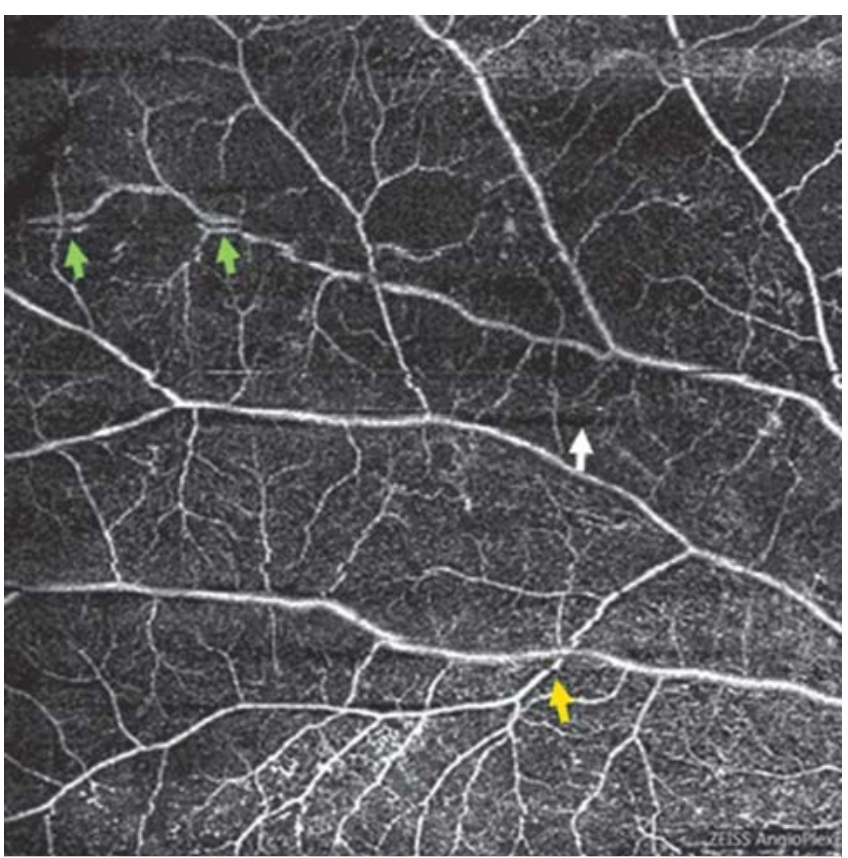

- Abb. 11 Bei mangelnder Kooperation des Patienten zeigen sich Bandenbildung (dunkle und helle Streifen) sowie Gefäßduplikationen (grüne Pfeile), Blinzelartefakte (weißer Pfeil) und Bewegungsartefakte (gelber Pfeil).

mit stark herabgesetztem Visus, schlechter Fixation oder schlechter Kooperation werden Bewegungsartefakte beobachtet.

\section{Blinzelartefakt (blink artifact)}

Auch durch Blinzeln des Patienten während des Scanvorgangs kann es zu Signalverlusten kommen. Diese stellen sich in den OCTA-Bildern aller Schichten von Netzhaut und Aderhaut als schwarze, senkrecht zur Scanrichtung verlaufende Linien an der gleichen Position dar ( $\bullet$ Abb. 11). Die Breite der Linien scheint dabei von der Dauer des Blinzelvorgangs abzuhängen. Je nach Aus- 

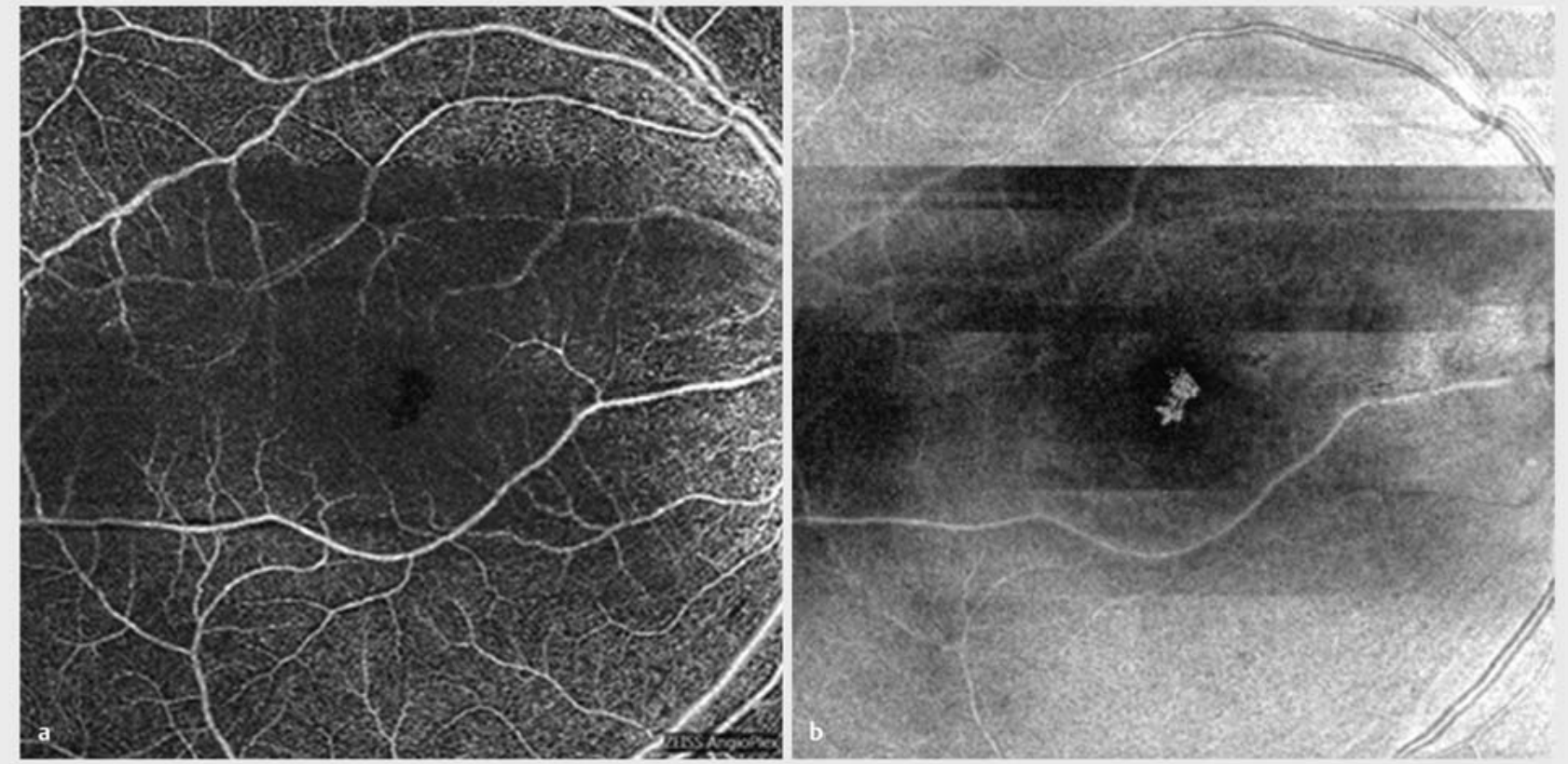

- Abb. 12 Bandenbildung mit breiten dunklen und hellen Streifen. a OCTA Retina, b En-Face-Struktur.

prägung kann dieses Artefakt eine Interpretation der OCTA-Aufnahmen erschweren. Bei Systemen mit Eye-Tracker ist eine starke Beeinträchtigung des OCT-Angiogramms jedoch selten. Auch bei forciertem Lidschluss entstehen lediglich wenige Blinzelartefakte.

\section{Bandenbildung (banding artifact)}

Verändert sich während des Aufnahmevorgangs der Abstand zwischen Gerät und Auge, so können direkt nebeneinander gelegene B-Scans eine unterschiedliche Helligkeit aufweisen. Dies kann im OCTA-Bild zu Bandenbildung führen, d.h. es ziehen sich breite horizontale Streifen mit unterschiedlicher Helligkeit über die OCTA-Aufnahmen sämtlicher Schichten der Netzhaut und Aderhaut ( $\triangleright$ Abb. 12 a, b).

Randduplikation oder Stretch-Artefakte (edge duplication/ stretching artifact)

Hierbei handelt es sich um kurze Streifen unterschiedlicher Helligkeit, die im Randbereich von OCTA-Aufnahmen auftreten können und dort eine Interpretation unmöglich machen. Hervorgerufen werden sie durch periodisch auftretenden Signalverlust, z.B. durch Mikrosakkaden.

\section{Diskussion}

Wie bereits in der Einleitung erläutert, erstellen bildgebende Verfahren idealerweise ein Abbild der Realität. Ein Auftreten von Bildartefakten ist systemimmanent und bei bildgebenden Verfahren jeder Art gegeben. Wie die Erfahrungen mit dieser neuen Methode zeigen, ist daher auch die OCTA erwartungsgemäß mit verschiedenen Artefakten behaftet - so wie grundsätzlich jedes bildgebende Verfahren. So treten auch bei der FAG (Fluoreszenz- angiografie), der Fundusfotografie und der multimodalen Bildgebung mit Laser-Scanning-Geräten und dem strukturellen OCT Artefakte auf. Bei diesen Verfahren kommt es z. B. zu Abschattungen durch Glaskörpertrübungen und -blutungen, während bei Aufnahmen mit Laser-Scanning-Geräten bereits ein trockenes Auge die Bildbeurteilung durch Artefakte erheblich beeinträchtigen kann. Der häufige Einsatz dieser etablierten Methode hat dazu geführt, dass die hier beschriebenen Artefakte gut bekannt sind und somit das Risiko einer Fehlinterpretation deutlich reduziert ist. Dementsprechend sind auch einige der hier im Zusammenhang mit der OCTA beschriebenen Artefakte den Anwendern des strukturellen OCT bereits geläufig.

Wie für die strukturelle OCT bereits beschrieben, scheint sich auch für die OCTA zu bestätigen, dass Artefakte grundsätzlich häufiger in Augen mit Pathologien zu beobachten sind als in gesunden Augen [8, 9, 11]. So zeigen Ergebnisse anderer Publikationen wie auch unsere Erfahrungen, dass z.B. Segmentierungsartefakte in erkrankten Augen häufiger auftreten als in gesunden Augen [11]. Dies ist auch zu erwarten, da die als Bezugsgröße zur automatischen Segmentierung erforderlichen Strukturen bei starken pathologischen Veränderungen von der Software nicht mehr zuverlässig erkannt werden können. Insbesondere bei ausgeprägten pathologischen Veränderungen der Netzhaut und Aderhaut sollten daher die Segmentierungslinien unbedingt immer auf Plausibilität überprüft werden, um Segmentierungsartefakte auszuschließen und eine zutreffende Bewertung sowie richtige Diagnose zu gewährleisten.

Während Bewegungsartefakte, Blinzelartefakte und Randduplikation deutlich als Störung der OCTA-Aufnahmen auszumachen sind und daher i.d.R. leicht als Artefakte zu identifizieren sind, können Segmentierungs-, Projektions- und Abschattungs- 
artefakte sowie Fenstereffekte dazu führen, dass auffällige Strukturen scheinbar in Schichten auftreten, in denen sie in der Realität nicht vorliegen. Insbesondere auf diese Artefakte sollte daher bei der Analyse der OCTA-Daten geachtet werden, um eine korrekte klinische Bewertung sicherzustellen.

So sollte etwa die Möglichkeit von Projektionsartefakten bei der Interpretation der OCTA-Daten grundsätzlich in Betracht gezogen werden. Wie auch von Spaide et al. bereits beschrieben, treten Projektionsartefakte vor allem in Bereichen mit darüber liegenden Gefäßen häufig auf [5]. Daher sollte durch eine Analyse des gesamten OCTA-Datensatzes kritisch geprüft werden, ob identische, charakteristische Gefäßmuster in mehreren Netzhautschichten wiederholt auftreten.

Grundsätzlich können sehr stark getrübte Medien die Interpretation der OCTA-Aufnahmen deutlich erschweren. Dies gilt allerdings ohnehin auch für andere bildgebende Verfahren wie etwa die FAG, bei der z. B. durch Glaskörperhämorrhagie ebenfalls die Interpretation der Aufnahmen meist erheblich erschwert ist.

Insgesamt bleibt festzuhalten, dass trotz Vorliegen diverser Artefakte doch in fast allen Fällen eine gute qualitative Beurteilung der OCTA-Aufnahmen möglich ist und ganz wesentliche Erkenntnisse zum Status verschiedener Erkrankungen des hinteren Augenabschnitts liefert.

Um OCTA-Aufnahmen zuverlässig und klinisch zutreffend zu bewerten, ist neben einem genauen Verständnis der Netzhautmorphologie auch ein gutes Verständnis über Entstehung und Auswirkungen von Bildartefakten außerordentlich wichtig. Die Interpretation erfordert wie bei allen anderen bildgebenden Verfahren der Netzhaut viel Erfahrung.

\section{Fazit}

Zusammenfassend lässt sich somit festhalten, dass bei der OCTA erwartungsgemäß wie bei jedem anderen bildgebenden Verfahren auch Artefakte auftreten. Dennoch lassen sich die OCTA-Aufnahmen bez. der Fragestellung qualitativ fast immer gut auswerten und liefern uns unverzichtbare Erkenntnisse zu Morphologie und Perfusionsstatus von Netzhaut und Aderhaut. Bei guter Kenntnis der möglichen Artefakte und entsprechender kritischer Datenanalyse ist eine korrekte Bewertung der OCTA-Aufnahmen gut möglich, um eine exakte klinische Diagnose zu stellen.

\section{Interessenkonflikt}

Finanzielle Unterstützung einschließlich Open-Access-Publikationsgebühr durch Carl Zeiss Meditec Vertriebsgesellschaft mbH, Oberkochen.

Literatur

[1] Lang GE, Enders C, Werner JU. Neue Möglichkeiten in der retinalen Diagnostik mittels OCT-Angiografie. Klin Monatsbl Augenheilkd 2016; 233: 613-621

[2] de Carlo TE, Romano A, Waheed NK et al. A review of optical coherence tomography angiography (OCTA). Int J Retina Vitreo 2015; 1: 5, eCollection 2015

[3] Yu S, Lu J, Cao D et al. The role of optical coherence tomography angiography in fundus vascular abnormalities. BMC Ophthalmol 2016; 16: 107
WICHTIGE ARTEFAKTE ERKENNEN -

EMPFEHLUNGEN FÜR DIE KLINISCHE PRAXIS

- Grundsätzlich sollte der gesamte OCT-/OCTA-Datensatz zur Bewertung herangezogen werden; eine sorgfältige und vergleichende Analyse auch unter Berücksichtigung der Anamnese, des ophthalmoskopischen Befunds und der Fundusaufnahme ist unerlässlich.

- Es sollte immer bedacht werden, dass Bereiche mit einem sehr langsamen oder schnellen Blutfluss (z. B. Mikroaneurysmen) in der OCTA nicht darstellbar sind.

- Der ausgewählte Segmentierungsbereich sollte immer auf Plausibilität geprüft und ggf. korrigiert werden. Ungenauigkeiten in der (automatischen) Segmentierung können die Ursache auffällig veränderter OCTA-Befunde sein.

- Treten identische, charakteristische Gefäßmuster in mehreren Netzhautschichten wiederholt auf, so kann es sich in einer Schicht um ein Projektionsartefakt handeln.

- Ist bekannt, dass eingetrübte Medien wie z. B. Glaskörpertrübungen, Glaskörperblutungen, mature Katarakt oder ein ausgeprägtes Ödem vorliegen (Fundusfotografie bzw. OCT-Schnittbild prüfen), sollte bei der Interpretation der OCTA-Aufnahmen auf mögliche Abschattungsartefakte in den Schichten geachtet werden, die unterhalb dieser Strukturen liegen.

- Bei Erkrankungen, die einen lokalen Ausfall bestimmter Netzhautschichten zur Folge haben (z. B. geografische Atrophie), ist bei der klinischen Bewertung der OCTA-Aufnahmen zu beachten, dass es in den darunter liegenden Schichten in diesen Bereichen zu einer Signalverstärkung kommen kann, die fälschlicherweise als Neovaskularisation interpretiert werden kann.

[4] Chen FK, Viljoen RD, Bukowska DM. Classification of image artefacts in optical coherence tomography angiography of the choroid in macular diseases. Clin Experiment Ophthalmol 2016; 44: 388-399

[5] Spaide RF, Fujimoto JG, Waheed NK. Image artifacts in optical coherence tomography angiography. Retina 2015; 35: 2163-2180

[6] Coscas G, Lupidi M, Coscas F. Image analysis of optical coherence tomography angiography. Dev Ophthalmol 2016; 56: 30-36

[7] Ghasemi Falavarjani K, Al-Sheik M, Akil H et al. Image artefact in sweptsource optical coherence tomography angiography. $\mathrm{Br}$ J Ophthalmol 2016; Jul 20. pii: bjophthalmol-2016-309104. doi: DOI: 10.1136/ bjophthalmol-2016-309104

[8] Song Y, Lee BR, Shin YW et al. Overcoming segmentation errors in measurements of macular thickness made by spectral domain optical coherence tomography. Retina 2012; 32: 569-580

[9] Asrani S, Essaid L, Alder BD et al. Artifacts in spectral-domain optical coherence tomography measurements in glaucoma. JAMA Ophthalmol 2014; 132: 396-402

[10] Zhang A, Zhang Q, Chen CL et al. Methods and algorithms for optical coherence tomography-based angiography: a review and comparison. J Biomed Opt 2015; 20: 100901. doi: DOI: 10.1117/1.JBO.20.10.100901

[11] Say EA, Ferenczy S, Magrath GN et al. Image quality and artifacts on optical coherence tomography angiography. Comparison of pathologic and paired fellow eyes in 65 patients with unilateral choroidal melanoma treated with plaque radiotherapy. Retina 2016; DOI: 10.1097/ IAE.0000000000001414 